\title{
Development of Caffeine Detection Using Taste Sensor with Lipid/Polymer Membranes
}

\author{
Haifeng Shen*, Masaaki Habara ${ }^{1}$ and Kiyoshi Toko \\ Graduate School of Information Science and Electrical Engineering, Kyushu University, \\ Fukuoka 819-0395, Japan \\ ${ }^{1}$ Graduate School of System Life Sciences, Kyushu University, Fukuoka 819-0395, Japan
}

(Received February 8, 2008; accepted June 3, 2008)

Key words: taste sensor, lipid/polymer membrane, caffeine

A multichannel taste sensor, i.e., an electronic tongue, with global selectivity is composed of several types of lipid/polymer membrane for transforming information on taste substances into electric signals that are input to a computer. However, the taste sensor has poorer sensitivity to uncharged molecules such as caffeine, a bitter substance, than to charged taste substances. In this study, we developed a taste sensor with higher sensitivity to detect caffeine using a taste sensor with lipid/polymer membranes that are formed with different lengths of alkyl chain of lipid, namely, tetra- $n$-octylammonium bromide (R8), tetrakis-(decyl)-ammonium bromide (R10), tetradodecylammonium bromide (TDAB; R12), and tetrahexadecylammonium bromide (R16). As a result, caffeine more preferably interacted with lipid membranes containing amine compounds than with phosphate lipid membranes. We also found that the electric responses of the lipid membranes to caffeine were associated with the length of alkyl chain of a lipid and amount concentration in the membrane. From these results, we can estimate the composition optimum for enhancing sensitivity to caffeine.

\section{Introduction}

A taste sensor transforms information on taste substances into electric signals. The electric signals obtained from the sensor are converted to a digital code by a digital voltmeter and transferred to a computer. ${ }^{(1)}$ Transducers of a multichannel taste sensor are composed of lipid membranes immobilized with a lipid/polymer. The difference in potential between a multichannel electrode and a $\mathrm{Ag} / \mathrm{AgCl}$ reference electrode is measured. The quality and intensity of the sample can be expressed graphically on a taste map in a two or three-dimensional space.

Caffeine is found in various quantities in beans, leaves and fruit of more than 60 plants, in which it acts as a natural pesticide that paralyzes and kills certain insects feeding on such plants. It is most commonly consumed by humans in infusions extracted

*Corresponding author: e-mail: Jacky@belab.ed.kyushu-u.ac.jp 
from coffee beans and tea leaves, as well as from various foods and drinks containing products derived from kola nuts or cacao. Caffeine is a xanthine alkaloid compound that acts as a psychoactive stimulant and diuretic in humans. It is an alkaloid classified into the purine group. Alkaloids are nitrogenous organic compounds of plant origin that have physiological actions on humans. ${ }^{(2)}$ A purine is a colorless crystalline compound with basic properties, forming uric acid on oxidation. Note from Fig. 1 that caffeine consists of a pyrimidine ring and an imidazole ring, and forms a heterocyclic aromatic organic compound. Caffeine also does not have the basic property of an electrolyte and is an uncharged molecule.

In our previous studies, the sensitivity of a taste sensor to many types of foodstuff such as wine, ${ }^{(3)}$ beer, ${ }^{(4-6)}$ milk, ${ }^{(7,8)}$ coffee, ${ }^{(9)}$ rice, ${ }^{(10)}$ and soybean paste ${ }^{(11)}$ was examined. However, the sensitivity of a taste sensor with a lipid/polymer membrane to molecules of uncharged taste substances is poorer than that to charged substances. Those studies were focused on enhancing sensitivity to uncharged taste substances.

Recently, using an analytical chemistry approach, binding and positioninginduced interaction have been observed between a membrane model composed of 1,2-dimyristelaidoyl-sn-glycero-3-phosphocholine (DMLPC) and caffeine. ${ }^{12)}$ DMLPC is a lipid that has an amine group and a phosphoric group with an alkane-like region. Interaction occurs between DMLPC and caffeine, for example, a strong interaction in the alkane-like region and a weak interaction at the amine group, whereas no interaction occurs around the phosphoric group. In our previous study, using a taste sensor with a series of lipid/polymer membranes, ${ }^{(13)}$ we demonstrated that a lipid membrane formed by an amine compound exhibits a higher sensitivity than that formed by a phosphoric compound. The aim of the present study is to determine the optimal composition of a lipid/polymer membrane in terms of the length of alkyl chains and the amount of the lipids to improve the sensitivity of caffeine detection.
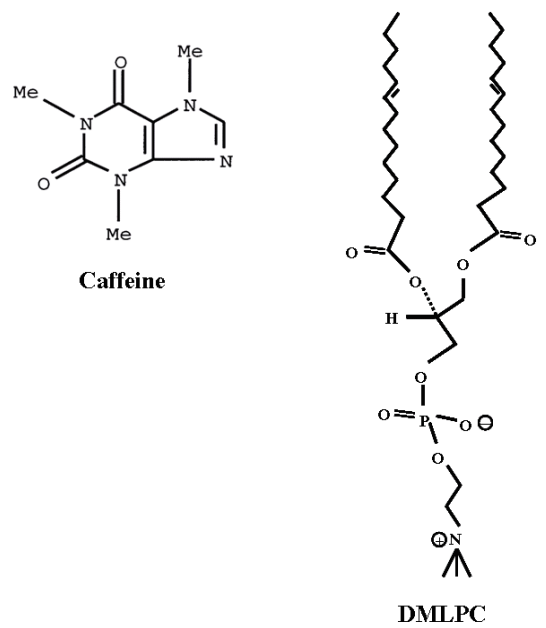

Fig. 1. Chemical structures of caffeine and 1,2-dimyristelaidoyl-sn-glycero-3-phosphocholine (DMLPC). 


\section{Materials and Methods}

Figure 2 shows a schematic illustration of the taste-sensing system. The detection part of the sensor usually consists of eight electrodes with different lipid/polymer membranes. The sensor detects taste information, which transformed into electric signals of membrane potentials. It is required to measure different taste substances with different lipid/polymer membranes. As shown in Fig. 3, the taste sensing system SA402B (Intelligent Sensor Technology, Inc.) was used in this study.

The measurements were performed five times by a rotation procedure comprising a round of measurements for all samples. Taste discrimination was carried out by using a computer recognizing the signals obtained from these taste sensors in the form of patterns, the so-called "pattern recognition." Figure 4 shows the measurement procedure using the taste sensor. First, the control solution was measured (denoted Vc1). The

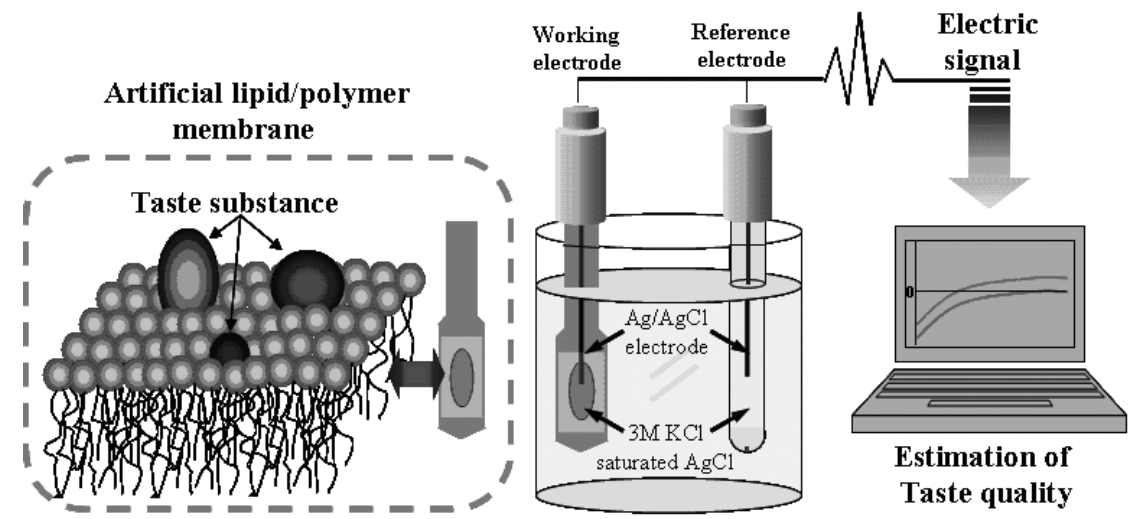

Fig. 2. Schematic illustration of taste sensor.

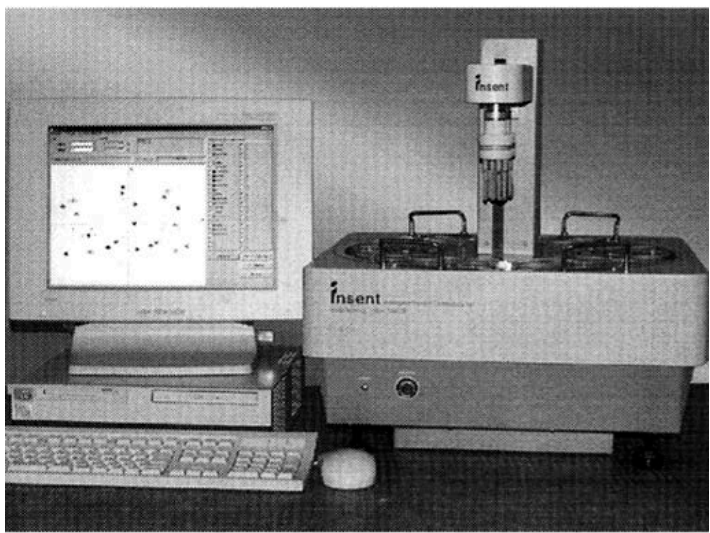

Fig. 3. Taste sensing system SA402B (Intelligent Sensor Technology, Inc.). 


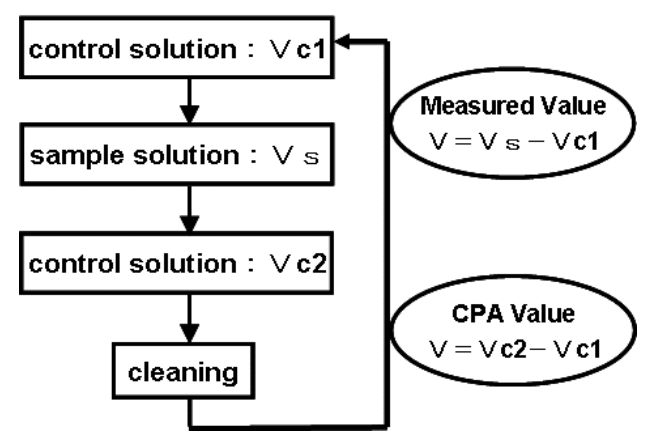

Fig. 4. Measurement procedure using taste sensor.

control solution had no taste and corresponds to saliva in humans. Then after the sample solution (Vs) was measured, the control solution was measured again. The obtained value was called Vc2. We calculated the difference between Vc1 and Vc2 (Vc2-Vc1). The difference is generally considered to be caused by changes in the charge density and structure of the membrane as a result of the adsorption of the taste substance. This difference (Vc2-Vc1) is called the "change in membrane potential caused by adsorption" (CPA).

Caffeine might interact with three functional groups of DMLPC, i.e., the alkane-like region, amine group, and phosphoric group. ${ }^{(12)}$ Therefore, simple-structure lipids were used in this study, that is, the structure of DMLPC was divided into small fragments expressing their functional group to control the sensitivity of caffeine. As shown in Table 1, dioctyl phosphate (DOP) and phosphoric di-n-decylester (2C10) were used as lipids with an alkyl chain and a phosphoric group that has a negative charge. Oleylamine (OAm), trioctylmethylammonium chloride (TOMA), and tetradodecylammonium bromide (TDAB) were used as amine lipids with alkyl chain that induce hydrophobic interaction, and have a positive charge. Moreover, Evanics and Prosser ${ }^{(12)}$ have reported that caffeine strongly interacts with DMLPC in the alkane-like region and weakly interacts at the amine group but not at the phosphoric group. Thus, we supposed that the adjustment of the hydrophobicity of the alkyl chain can enhance caffeine responses to the lipid membrane. Thus, to improve the sensitivity of caffeine detection, the length of alkyl chains were investigated using tetra-n-octylammonium bromide (R8), tetrakis-(decyl)-ammonium bromide (R10), and tetrahexadecylammonium bromide (R16).

Caffeine was obtained from Kanto Chemical. Co., Inc. (Tokyo, Japan) and used without further purification. The lipids listed in Table 1 were used for preparing the membranes in this study. DOP, 2C10, OAm, and TOMA were obtained as solutions, from Tokyo Chemical Industry Co., Ltd. (Tokyo, Japan) and TDAB was obtained from Sigma-Aldrich Japan K.K. (Tokyo, Japan). R8, R10, and R16 were purchased from Fluka Chemie Co., Ltd. (Tokyo, Japan). Each lipid was mixed in a test tube with 800 mg of polyvinyl chloride (PVC) and a plasticizer (2-nitrophenyl octyl ether, $1.0 \mathrm{ml}$ ) dissolved simultaneously in tetrahydroguran. One $\mathrm{h}$ of gentle vortexing was usually sufficient to obtain a clear mixture. Successively, the mixture was dried on a glass plate, 
Table 1

Materials used for membrane formation.

\begin{tabular}{lll}
\hline & Material & Abbr. \\
\hline \multirow{2}{*}{ Phosphoric group } & Dioctyl phosphate & DOP \\
& Di-n-decylester & 2C10 \\
\hline \multirow{3}{*}{ N-H group } & Oleylamine & OAm \\
& Trioctylmethylammonium chloride & TOMA \\
& Tetradodecylammonium bromide & TDAB \\
\hline & 2-Nitrophenyl octyl ether & NPOE \\
& Polyvinyl chloride & PVC \\
\hline
\end{tabular}

which was placed on a hot plate maintained at approximately $30^{\circ} \mathrm{C}$. The lipid/polymer membrane thus prepared was a transparent, colorless, soft film of approximately $200 \mu \mathrm{M}$ thickness.

Each electrode consisted of a Ag wire whose surface was plated with $\mathrm{AgCl}$, and the internal cavity was filled with $3 \mathrm{M} \mathrm{KCl}$ and saturated $\mathrm{AgCl}$ solution (Fig. 2). The membranes were preconditioned in $30 \mathrm{mM} \mathrm{KCl}$ and $0.3 \mathrm{mM}$ tartaric acid for $48 \mathrm{~h}$.

\section{Results and Discussion}

As shown in Fig. 5, electric responses to caffeine were measured individually for membranes composed of $0.1 \mu \mathrm{M}$ each of DOP, 2C10, OAm, TOMA, and TDAB. As a result, the membrane containing DOP or $2 \mathrm{C} 10$, which has the phosphoric group, showed weak responses to $10 \mathrm{mM}$ caffeine. In contrast, the membrane formed with 0.1 $\mu \mathrm{M}$ OAm, TOMA, or TDAB exhibited excellent responses to caffeine, indicating that amine compounds such as OAm, TOMA, and TDAB can possibly increase sensitivity to caffeine. This finding was demonstrated partly in our previous study. ${ }^{(13)}$ In particular, the highest sensitivity to caffeine was observed when the membrane composed of TDAB was used. Therefore, in this study, we examined the relationship between alkyl chain length and sensitivity for caffeine detection, we used a series of lipid membranes formed with different alkyl chain lengths based on the structure of TDAB (R12), i.e., R8, R10, and R16. The chemical structure of each lipid is shown in Fig. 6.

Figure 7 shows the electric responses of lipid membranes with different alkyl chain lengths to caffeine. The lateral axis is the alkyl chain length of lipids contained in membranes, and the vertical axis is the electric responses to various concentrations of caffeine $(0.1-10 \mathrm{mM})$. As a result, the responses of the membrane composed lipids (R8-R16) at each amount to caffeine, except $30 \mu \mathrm{M}$, showed a caffeine concentration dependence indicating that amine compounds induce a unique sensitivity to caffeine. This finding is supported by our previous study using membranes composed of amine compounds such as TOMA and TDAB, in which a caffeine concentration dependence was also observed. ${ }^{(13)}$

Furthermore, the electric responses to caffeine were compared at different concentrations $(0.03-30 \mu \mathrm{M})$ of each lipid (R8-R16). We observed that the membrane formed with $0.03 \mu \mathrm{M}$ or $0.3 \mu \mathrm{M}$ R10 showed a high-level response to caffeine compared 


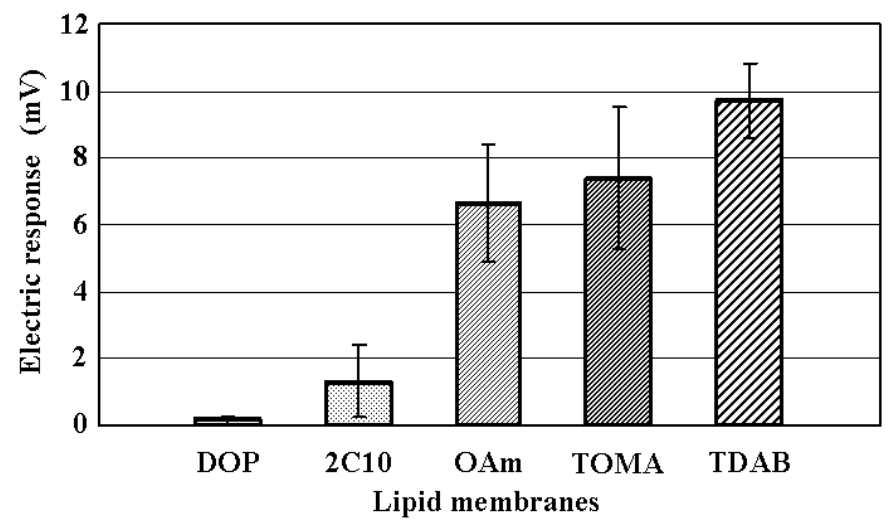

Fig. 5. Electric responses of lipid membranes containing $0.1 \mu \mathrm{M}$ each of DOP, 2C10, OAm, TOMA, and TDAB to caffeine.

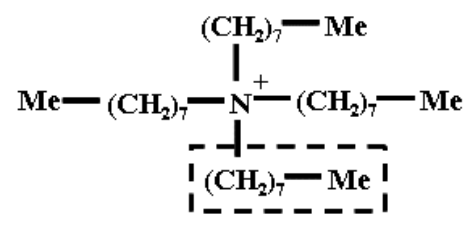

(a) Tetra-n-octylammonium bromide (R8)

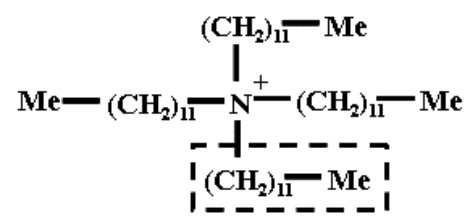

(c) Tetradodecylammonium bromide (R12; TDAB)

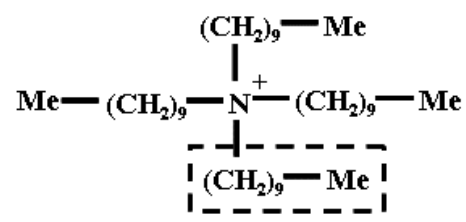

(b) Tetrakis-(decyl)-ammonium bromide (R10)

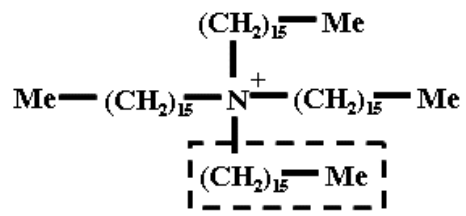

(d) Tetrahexadecylammonium bromide (R16)

Fig. 6. Chemical structures of four types of lipid with different alkyl chain lengths (R8-R16).

with the other membranes (Figs. 7(a) and 7(b)). Moreover, a high-level response was obtained for the membrane containing $3 \mu \mathrm{M}$ R8 or $3 \mu \mathrm{M}$ R16 (Fig. 7(c)), whereas the response disappeared for the membrane formed with each lipid at $30 \mu \mathrm{M}$ (Fig. 7(d)). For detecting the nonelectrolyte taste substances, such as caffeine, the substance adsorbs onto the surface of the membrane and may increase the packing density of 


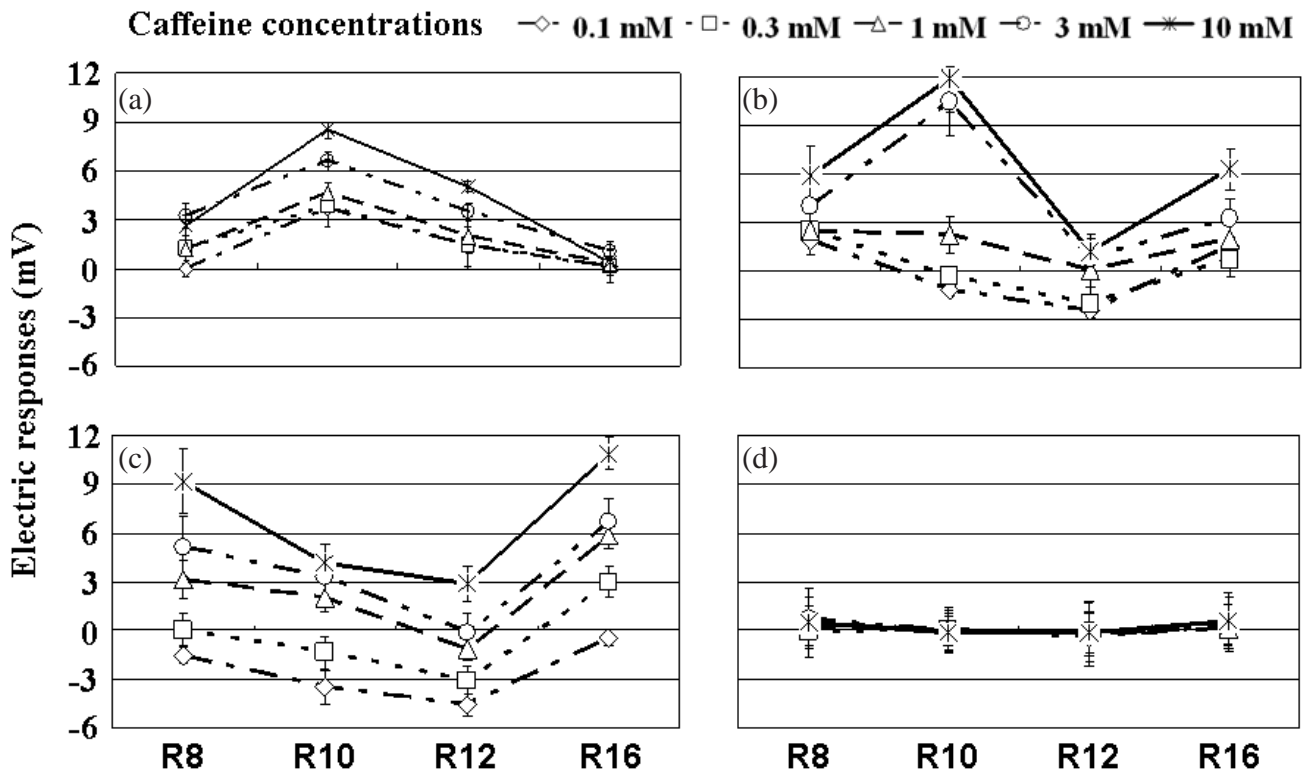

Fig. 7. Electric responses of lipid membranes containing different alkyl chain lengths to caffeine. (a)(d) show the concentration of each lipid in the membranes (i.e., (a) $0.03 \mu \mathrm{M}$; (b) $0.3 \mu \mathrm{M}$; (c) $3 \mu \mathrm{M}$; (d) $30 \mu \mathrm{M})$.

Table 2

Optimum concentrations of lipids with different alkyl chain lengths for caffeine responses.

\begin{tabular}{lccc}
\hline Concentration $(\mu \mathrm{M})$ & $0.03-0.3$ & 3 & 30 \\
\hline Alkyl chain length & R10 & R8 or R16 & None \\
\hline
\end{tabular}

the membrane equivalently or block ion permeation. It causes the suppression of ion permeation through the membrane.(1) Thus, we can observe the change of potential using the taste sensor. To enhance the ion permeation of the membrane, we also speculated the optimum concentrations of lipids in the membrane. In this study, we found a higher electric response ( $>9 \mathrm{mV}$ ) at $0.3 \mu \mathrm{M}$ for $\mathrm{R} 10$ and at $3 \mu \mathrm{M}$ for R 8 and R16. From these results, to obtain a higher sensitivity of caffeine detection, the optimum concentrations of lipids contained in membranes used as summarized in Table 2. All of these suggest that the concentrations of lipids in membranes are another important factor for enhancing responses to caffeine.

\section{Conclusions}

Caffeine is an alkaloid, which is a type of nitrogenous organic compound, and has no basic property and electric charge. For a taste sensor, lipid/polymer membranes 
are required to be sufficiently sensitive to such nonelectrolytes. In our previous study, a higher sensitivity was observed for a taste sensor with lipid/polymer membranes consisting of amine compounds but not phosphoric compounds. ${ }^{(12)}$ In this study, we observed that caffeine more preferably interacted with membranes containing amine compounds than with membranes containing phosphate lipids. In addition, we speculated that an alteration in the hydrophobic interaction between caffeine and alkyl chains of lipid might induce an alteration of the interaction between caffeine and amine groups, influencing the electric responses. We also proposed an optimum concentration of lipids with different alkyl chain lengths contained in membranes for obtaining a higher sensitivity to caffeine. Because alkaloids interact with biological materials with a hydrophobic property, we can predict the importance of alkyl chain length and lipid concentration in membranes to improve the sensitivity to taste substances with uncharged molecules such as caffeine. Thus, the detailed mechanism underlying caffeine-induced potential changes in response to the lipid/polymer needs to be clarified in future studies.

\section{References}

1 K. Toko: Biomimetic Sensor Technology (Cambridge University Press, Cambridge, 2000).

2 A. L. Anaya, R. Cruz-Ortega and G. R. Waller: Front. Biosci. 11 (2006) 2354.

3 S. Baldacci, T. Matsuno, K. Toko, R. Stella and D. De Rossi: Sens. Mater. 10 (1995) 185.

4 K. Toko, T. Matsuno, K. Yamafuji, K. Hayashi, H. Ikezaki, K. Sato, R. Toukubo and S. Kawarai: Biosens. Bioelectron. 9 (1994) 359.

5 H. Ikezaki, K. Hayashi, M. Yamanaka, R. Tatsukawa, K. Toko and K. Yamafuji: Trans. IEICE Jpn. J74-C-II (1991) 434 (in Japanese).

6 S. Ezaki, T. Yuki, K. Toko, Y. Tsuda and K. Nakatani: Trans. IEE Jpn. 117-E (1997) 449 (in Japanese).

7 K. Toko, T. Iyota, Y. Mizota, T. Matsuno, T. Yoshioka, T. Doi, S. Iiyama, T. Kato, K. Yamafuji and R. Watanabe: Jpn. J. Appl. Phys. 34 (1995) 6278.

8 H. Yamada, Y. Mizota, K. Toko and T. Doi: Mater. Sci. Eng., C 5 (1997) 41.

9 T. Fukunaga, K. Toko, H. Mori, T. Nakabayashi and M. Kanda: Sens. Mater. 8 (1996) 47.

10 M. Yahiro, K. Toko and S. Iiyama: Trans. IEE Jpn. 117-E (1997) 187 (in Japanese).

11 T. Imamura, K. Toko, S. Yanagisawa and T. Kume: Sens. Actuators, B 37 (1996) 179.

12 F. Evancis, R. S. Prosser: Anal. Chim. Acta 534 (2005) 21.

13 M. Habara, H. F. Shen, K. Toko: Research Reports on Information Science and Electrical Engineering of Kyushu University 11 (2006) 97. 